\title{
On the meromorphic solutions of generalized $q$-difference equations
}

\author{
Hongqiang $\mathrm{Tu}^{\mathrm{a}}$, Yongyi Gu $\mathrm{u}^{\mathrm{b}, *}$ \\ a School of Mathematics and Information Science, Guangzhou University, Guangzhou 510006 China \\ b Big Data and Educational Statistics Application Laboratory, Guangdong University of Finance and \\ Economics, Guangzhou 510320 China
}

*Corresponding author, e-mail: gdguyongyi@163.com

Received 2 Apr 2020

Accepted 18 Aug 2020

ABSTRACT: A generalized $q$-difference analogue of the lemma on the logarithmic derivative is introduced, which is an improvement of previous result due to Korhonen, and was applied to the growth of meromorphic solutions of some linear difference equations. Examples are given to illustrate the sharpness of the results.

KEYWORDS: meromorphic solutions, q-difference equations, growth

MSC2010: 30D35 39B32 39A10

\section{INTRODUCTION}

When applying Nevanlinna theory to the growth and value distribution of meromorphic solutions of differential equations in the complex plane, estimates involving logarithmic derivatives have often proved useful, see $[1,2]$. A difference analogue of the lemma on the logarithmic derivative for finiteorder meromorphic functions in $\mathbb{C}$ was obtained independently by Halburd and Korhonen [3, 4] and Chiang and Feng [5]. Halburd et al [6] showed that if the hyper-order $\sigma_{2}(f)$ of $f$ satisfies $\sigma_{2}(f)=\sigma_{2}<$ 1 and $\varepsilon>0$, then

$$
m\left(r, \frac{f(z+c)}{f(z)}\right)=o\left(\frac{T(r, f)}{r^{1-\sigma_{2}-\varepsilon}}\right)
$$

for all $r$ outside a set of finite logarithmic measure.

Here it is assumed that the reader is familiar with Nevanlinna theory, see $[7,8]$. In what follows, the growth order, the type and the exponent of convergence of the poles of a meromorphic function $f(z)$ are represented by $\sigma(f), \tau(f)$ and $\lambda\left(\frac{1}{f}\right)$, respectively. For $p \in \mathbb{N}$, we define the iterated order, and the iterated type of $f(z)$, respectively, as follows:

$$
\begin{aligned}
& \sigma_{p}(f)=\limsup _{r \rightarrow \infty} \frac{\log _{p} T(r, f)}{\log r}, \\
& \tau_{p}(f)=\limsup _{r \rightarrow \infty} \frac{\log _{p-1} T(r, f)}{r^{\sigma_{p}(f)}},
\end{aligned}
$$

see [9]. In particular, $\sigma_{1}(f)=\sigma(f)$ is the order, $\tau_{1}(f)=\tau(f)$ is the type, and $\sigma_{2}(f)$ is the hyperorder of $f$. By a meromorphic function we always mean a function meromorphic in $\mathbb{C}$.

Korhonen [10] obtained a generalization of (1) by proving that if $\omega(z)=c z^{n}+p_{n-1} z^{n-1}+\cdots+p_{0}$ and $\varphi(z)=c z^{n}+q_{n-1} z^{n-1}+\cdots+q_{0}$ are non-constant polynomials, then for any non-rational meromorphic function $f$ of hyper-order less than $\frac{1}{n^{2}}$,

$$
m\left(r, \frac{f \circ \omega}{f \circ \varphi}\right)=o\left(T\left(|c| r^{n}, f\right)\right)
$$

as $r \rightarrow \infty$ outside an exceptional set of finite logarithmic measure.

The purpose of this paper is to improve the estimate (2) by giving an explicit error term.

Theorem 1 Let $f$ be a non-rational meromorphic function, let $\omega(z)=c z^{n}+p_{n-1} z^{n-1}+\cdots+p_{0}$ and $\varphi(z)=c z^{n}+q_{n-1} z^{n-1}+\cdots+q_{0}$ be non-constant polynomials. If $\sigma_{2}(f)=\sigma_{2}<\frac{1}{n^{2}}$ and $\varepsilon>0$, then

$$
m\left(r, \frac{f \circ \omega}{f \circ \varphi}\right)=o\left(\frac{T\left(|c| r^{n}, f\right)}{r^{\frac{1}{n}-n \sigma_{2}-\varepsilon}}\right)
$$

for all $r$ outside an exceptional set of finite logarithmic measure.

Remark 1 Theorem 1 is a generalization of Theorem 5.1 in [6] for meromorphic functions composed with polynomials.

Theorem 1 is a useful tool in the growth of finite-order transcendental meromorphic solutions 
of functional equations with polynomial compositions. In the remainder of this paper, we will apply it to the growth of meromorphic solutions of linear difference equations in the homogeneous and the non-homogeneous cases, when there is a dominating coefficient.

\section{APPLICATIONS TO FUNCTIONAL EQUATIONS}

By the use of recent new difference versions of Nevanlinna theory, many studies $[1,5,11-20]$ of the properties of meromorphic solutions of the difference equation

$a_{k}(z) f\left(z+c_{k}\right)+\cdots+a_{0}(z) f(z)=0, \quad k \in \mathbb{N}$,

and its special case $c_{j}=j, j=1, \ldots, k$, where $c_{j}$ are distinct non-zero complex constants and $a_{j}(z)$ are meromorphic functions, have appeared during recent years. Chiang and Feng [5] showed that if $a_{j}(z)(j=0,1, \ldots, k)$ are entire functions and if there exists an integer $l \in\{0, \ldots, k\}$ such that $\sigma\left(a_{l}\right)>$ $\max _{0 \leqslant j \leqslant k, j \neq l}\left\{\sigma\left(a_{j}\right)\right\}$, then every meromorphic solution $f(\equiv 0)$ of (3) with $c_{j}=j$ satisfies $\sigma(f) \geqslant$ $\sigma\left(a_{l}\right)+1$. In the case when there is more than one coefficients having the maximal order, Laine and Yang [11] proved that if $a_{j}(z)(j=0,1, \ldots, k)$ are entire functions of finite order such that among those having the maximal order $\sigma=\max _{0 \leqslant j \leqslant k}\left\{\sigma\left(a_{j}\right)\right\}$, exactly one has its type strictly greater than the others, then for every meromorphic solution $f(\not \equiv 0)$ of (3), we have $\sigma(f) \geqslant \sigma+1$.

Liu and Qi [16] investigated the growth of meromorphic solutions of linear $q$-difference equation

$$
a_{k}(z) f\left(q_{k} z+\eta_{k}\right)+\cdots+a_{0}(z) f\left(q_{0} z+\eta_{0}\right)=0
$$

where $\eta_{0}, \ldots, \eta_{k}$ are non-zero complex constants.

Assuming that $P_{i}(z)(i=0,1, \ldots, k)$ are polynomials with degree $n(\geqslant 1)$, we consider the equation

$$
a_{k}(z) f\left(P_{k}(z)\right)+\cdots+a_{0}(z) f\left(P_{0}(z)\right)=0,
$$

which contains (3) and (4) as special cases. When (5) has only one dominating coefficient and all coefficients are entire functions, we obtain the following result.

Theorem 2 Let $P_{j}(z)=c z^{n}+c_{j_{n-1}} z^{n-1}+\ldots+c_{j_{0}}(c \neq$ $0), j=0,1, \ldots, k$, be polynomials with degree $n \geqslant 1$ and let $H$ be a complex set satisfying $\overline{\operatorname{dens}}\{r=|z|$ : $z \in H\}>0$, and let $a_{j}(z), j=0,1, \ldots, k$ be entire functions satisfying $\max _{0 \leqslant j \leqslant k}\left\{\sigma\left(a_{j}\right)\right\} \leqslant \sigma$. If there exists an integer $l \in\{0, \ldots, k\}$ such that for some constants $0 \leqslant \alpha<\beta$ and $\delta>0$ sufficiently small,

$$
m\left(r, a_{l}(z)\right) \geqslant \beta r^{\sigma-\delta}, \quad m\left(r, a_{j}(z)\right) \leqslant \alpha r^{\sigma-\delta}
$$

for all $j=0,1, \ldots, k, j \neq l$, as $z \rightarrow \infty$ for $z \in H$, then every transcendental meromorphic solution $f(z)(\equiv 0)$ of (5) satisfies $\sigma(f) \geqslant \sigma\left(a_{l}\right) / n+1 / n^{2}$.

Note that in Theorem 2, the condition that there exists more than one coefficient having the maximal order and (5) has only one dominating coefficient. When there is only one dominating coefficient and all coefficients are meromorphic functions in (5), we obtain the following result.

Theorem 3 Let $P_{j}(z)=c z^{n}+c_{j_{n-1}} z^{n-1}+\ldots+c_{j_{0}}(c \neq$ $0), j=0,1, \ldots, k$, be polynomials with degree $n \geqslant 1$ and $a_{j}(z), j=0,1, \ldots, k$ be meromorphic functions. If there exists an integer $l \in\{0, \ldots, k\}$ such that $a_{l}(z)$ satisfies

$$
\begin{gathered}
\lambda\left(\frac{1}{a_{l}}\right)<\sigma\left(a_{l}\right)<\infty, \\
\max \left\{\sigma\left(a_{j}\right): j=0,1, \ldots, k, j \neq l\right\} \leqslant \sigma\left(a_{l}\right), \\
\sum_{\sigma\left(a_{j}\right)=\sigma\left(a_{l}\right), j \neq l} \tau\left(a_{j}\right)<\tau\left(a_{l}\right)<\infty,
\end{gathered}
$$

then every transcendental meromorphic solution $f(z)(\not \equiv 0)$ of $(5)$ satisfies $\sigma(f) \geqslant \sigma\left(a_{l}\right) / n+1 / n^{2}$.

Note that in Theorem 3, the condition that there exists more than one coefficient having the maximal order and (5) has only one coefficient having the maximal type.

Finally, we consider the case of the nonhomogeneous equation

$$
a_{k}(z) f\left(P_{k}(z)\right)+\cdots+a_{0}(z) f\left(P_{0}(z)\right)=F(z),
$$

where $P_{i}(z)(i=0,1, \ldots, k)$ are non-constant polynomials with different leading coefficients.

Theorem 4 Let $P_{j}(z)=c_{j p_{j}} z^{p_{j}}+c_{j p_{j}-1} z^{p_{j}-1}+\ldots+$ $c_{j 0}\left(c_{j p_{j}} \neq 0\right), j=0,1, \ldots, k$, be polynomials with degree $p_{j} \geqslant 1$, let $a_{j}(z), j=0,1, \ldots, k, F(z)$ be meromorphic functions, and let $l$ satisfy the conditions of Theorem 3. We have

(i) if $\sigma(F)<\sigma\left(a_{l}\right)$, or $\sigma(F)=\sigma\left(a_{l}\right)$ and $\sum_{\sigma\left(a_{j}\right)=\sigma\left(a_{l}\right), j \neq l} \tau\left(a_{j}\right)+\tau(F)<\tau\left(a_{l}\right), \quad$ or $\sigma(F)=\sigma\left(a_{l}\right)$ and $\sum_{\sigma\left(a_{j}\right)=\sigma\left(a_{l}\right)} \tau\left(a_{j}\right)<\tau(F)$, then every meromorphic solution $f(z)(\equiv 0)$ of (6) satisfies $\sigma(f) \geqslant \frac{\sigma\left(a_{l}\right)}{\max \left\{p_{j}\right\}}$; 
(ii) if $F(z) \equiv 0$, then every meromorphic solution $f(z)(\not \equiv 0)$ of (6) satisfies $\sigma(f) \geqslant \frac{\sigma\left(a_{l}\right)}{\max \left\{p_{j}\right\}}$;

(iii) if $\sigma(F)>\sigma\left(a_{l}\right)$, then every meromorphic solution $f(z)(\equiv 0)$ of $(6)$ satisfies $\sigma(f) \geqslant \frac{\sigma(F)}{\max \left\{p_{j}\right\}}$.

Comparing Theorem 4 to Theorem 2 and Theorem $3, P_{j}(z)(j=0,1, \ldots, k)$ are non-constant polynomials with different leading coefficients in (6).

\section{EXAMPLES}

The following Example 1 is related to the sharpness of Theorem 1.

Example 1 The functions $f(z)=\mathrm{e}^{z}, \omega(z)=z^{2}+z+$ 1 and $\varphi(z)=z^{2}$ satisfy the hypothesis of Theorem 1 . Now $\frac{T\left(r^{2}, f\right)}{r^{\frac{1}{2}-\varepsilon}}=\frac{r^{\frac{3}{2}+\varepsilon}}{\pi}$ and

$m\left(r, \frac{f \circ \omega}{f \circ \varphi}\right)=m\left(r, \mathrm{e}^{z+1}\right)=\frac{r}{\pi}+O(1)=o\left(\frac{T\left(r^{2}, f\right)}{r^{\frac{1}{2}-\varepsilon}}\right)$.

The following Example 2 shows that the strict inequality $\sigma(f)>\sigma\left(a_{l}\right) / n+1 / n^{2}$ can occur in Theorem 2 .

Example 2 For Theorem 2, $f(z)=\mathrm{e}^{z}$ satisfies the difference equation

$\mathrm{e}^{2 z} f\left(3 z^{2}-2 z+1\right)+\mathrm{e}^{z} f\left(3 z^{2}-z+1\right)-2 f\left(3 z^{2}+1\right)=0$,

where $a_{2}(z)=\mathrm{e}^{2 z}, a_{1}(z)=\mathrm{e}^{z}, a_{0}(z)=-2$ and $H=$ $\{z: \arg z=0\}$. Clearly, $\sigma\left(a_{2}\right)=\sigma\left(a_{1}\right)=1, \sigma\left(a_{0}\right)=$ 0 and $l=n=2$. Here $\sigma(f)=1>\frac{3}{4}=\frac{1}{2}+\frac{1}{2^{2}}$.

The following Examples 3 and 4 show that the equality $\sigma(f)=\sigma\left(a_{l}\right) / n+1 / n^{2}$ can occur in Theorems 2 and 3.

Example 3 For Theorem 2, $f(z)=\mathrm{e}^{-z^{2}}$ satisfies the difference equation

$$
\mathrm{e}^{z+1} f(z+1)-\mathrm{e}^{-z} f(z)=0,
$$

where $a_{1}(z)=\mathrm{e}^{z+1}, a_{0}(z)=-\mathrm{e}^{-z}$ and $H=\{z$ : $\arg z=0\}$. Then $l=n=1$ and $\sigma\left(a_{1}\right)=\sigma\left(a_{0}\right)=1$, we have $\sigma(f)=2=\sigma\left(a_{1}\right)+1$.

Example 4 For Theorem 3, $f(z)=\mathrm{e}^{z^{3}} \tan z$ satisfies the difference equation

$$
a_{2}(z) f\left(z+\frac{3}{2} \pi\right)+a_{1}(z) f(z+\pi)+a_{0}(z) f(z)=0,
$$

where $a_{2}(z)=\mathrm{e}^{-\left(\frac{9}{2} \pi z^{2}+\frac{27}{4} \pi^{2} z+\frac{27}{8} \pi^{3}\right)} \tan ^{2} z, a_{1}(z)=$ $2 \mathrm{e}^{-\left(3 \pi z^{2}+3 \pi^{2} z+\pi^{3}\right)}$ and $a_{0}(z)=-1$. Then, $a_{j}(z), j=$ $0,1,2$ satisfy

$$
\begin{gathered}
\lambda\left(\frac{1}{a_{2}}\right)=1<2=\sigma\left(a_{2}\right), \\
\max \left\{\sigma\left(a_{0}\right), \sigma\left(a_{1}\right)\right\}=2=\sigma\left(a_{2}\right), \\
\tau\left(a_{1}\right)=3<\frac{9}{2}=\tau\left(a_{2}\right),
\end{gathered}
$$

where $l=n=1$. Here $\sigma(f)=3=\sigma\left(a_{2}\right)+1$.

The following Example 5 shows that the result in Theorem 4 is sharp.

Example 5 For Theorem 4, we consider the meromorphic functions

$$
f(z)=\mathrm{e}^{3 z^{2}} \tan z \text { and } g(z)=\mathrm{e}^{z^{3}} \tan z .
$$

Case 1. If $\sigma(F)<\sigma\left(a_{l}\right)$ and $F(z)(\not \equiv 0)$, then $f(z)$ satisfies the difference equation

$$
a_{2}(z) f(z+3 \pi)+a_{1}(z) f(z+2 \pi)+a_{0}(z) f(z)=F(z),(7)
$$

where $a_{2}(z)=\mathrm{e}^{-3 z^{2}} \cot z, a_{1}(z)=\mathrm{e}^{z^{2}-12 \pi z-12 \pi^{2}}$, $a_{0}(z)=-\mathrm{e}^{z^{2}}$ and $F(z)=\mathrm{e}^{18 \pi z+27 \pi^{2}}$. Then, $a_{j}(z)$, $j=0,1,2$ and $F(z)$ satisfy

$$
\begin{gathered}
\lambda\left(\frac{1}{a_{2}}\right)=1<2=\sigma\left(a_{2}\right), \\
\sigma(F)=1<2=\max \left\{\sigma\left(a_{0}\right), \sigma\left(a_{1}\right)\right\}=\sigma\left(a_{2}\right), \\
\tau\left(a_{0}\right)+\tau\left(a_{1}\right)=\frac{1}{\pi}+\frac{1}{\pi}=\frac{2}{\pi}<\frac{3}{\pi}=\tau\left(a_{2}\right),
\end{gathered}
$$

where $l=2$ and $\max \left\{p_{2}, p_{1}, p_{0}\right\}=1$. Here $\sigma(f)=$ $\sigma\left(a_{2}\right)=2$.

Case 2. If $\sigma(F)=\sigma\left(a_{l}\right)$ and $\sum_{\sigma\left(a_{j}\right)=\sigma\left(a_{l}\right), j \neq l} \tau\left(a_{j}\right)+$ $\tau(F)<\tau\left(a_{l}\right)$, then $f(z)$ satisfies (7), where $a_{2}(z)=$ $\mathrm{e}^{-\left(7 z^{2}+18 \pi z+27 \pi^{2}\right)} \cot z, a_{1}(z)=\mathrm{e}^{z^{2}-12 \pi z-12 \pi^{2}}, a_{0}(z)=$ $-\mathrm{e}^{z^{2}}$ and $F(z)=\mathrm{e}^{-4 z^{2}}$. Then, $a_{j}(z), j=0,1,2$ and $F(z)$ satisfy

$$
\begin{gathered}
\lambda\left(\frac{1}{a_{2}}\right)=1<2=\sigma\left(a_{2}\right), \\
\sigma(F)=2=\max \left\{\sigma\left(a_{0}\right), \sigma\left(a_{1}\right)\right\}=\sigma\left(a_{2}\right), \\
\tau\left(a_{0}\right)+\tau\left(a_{1}\right)+\tau(F)=\frac{1}{\pi}+\frac{1}{\pi}+\frac{4}{\pi}=\frac{6}{\pi}<\frac{7}{\pi}=\tau\left(a_{2}\right),
\end{gathered}
$$

where $l=2$ and $\max \left\{p_{2}, p_{1}, p_{0}\right\}=1$. Here $\sigma(f)=$ $\sigma\left(a_{2}\right)=2$.

Case 3. If $\sigma(F)=\sigma\left(a_{l}\right)$ and $\sum_{\sigma\left(a_{j}\right)=\sigma\left(a_{l}\right), j \neq l} \tau\left(a_{j}\right)+$ $\tau\left(a_{l}\right)<\tau(F)$, then $f(z)$ satisfies (7), where $a_{2}(z)=$ $\mathrm{e}^{z^{2}-18 \pi z-27 \pi^{2}} \cot z, a_{1}(z)=\mathrm{e}^{-\left(z^{2}+12 \pi z+12 \pi^{2}\right)}, a_{0}(z)=$ $-\mathrm{e}^{-z^{2}}, F(z)=\mathrm{e}^{4 z^{2}}$. Then, $a_{j}(z), j=0,1,2$ and $F(z)$ satisfy

$$
\begin{gathered}
\lambda\left(\frac{1}{a_{2}}\right)=1<2=\sigma\left(a_{2}\right), \\
\sigma(F)=2=\max \left\{\sigma\left(a_{0}\right), \sigma\left(a_{1}\right)\right\}=\sigma\left(a_{2}\right), \\
\tau\left(a_{0}\right)+\tau\left(a_{1}\right)+\tau\left(a_{2}\right)=\frac{1}{\pi}+\frac{1}{\pi}+\frac{1}{\pi}=\frac{3}{\pi}<\frac{4}{\pi}=\tau(F),
\end{gathered}
$$


where $l=2$ and $\max \left\{p_{2}, p_{1}, p_{0}\right\}=1$. Here $\sigma(f)=$ $\sigma\left(a_{2}\right)=2$.

Case 4. If $F(z) \equiv 0$, then $g(z)$ satisfies the difference equation

$$
a_{2}(z) g\left(z+\frac{5 \pi}{2}\right)+a_{1}(z) g(z+2 \pi)+a_{0}(z) g(z)=0,
$$

where $a_{2}(z)=\mathrm{e}^{-\left(\frac{15 \pi}{2} z^{2}+\frac{75 \pi^{2}}{4} z+\frac{125 \pi^{3}}{8}\right)} \tan ^{2} z, a_{1}(z)=$ $2 \mathrm{e}^{-\left(6 \pi z^{2}+12 \pi^{2} z+8 \pi^{3}\right)}$ and $a_{0}(z)=-1$. Then, $a_{j}(z)$, $j=0,1,2$ satisfy

$$
\begin{gathered}
\lambda\left(\frac{1}{a_{2}}\right)=1<2=\sigma\left(a_{2}\right), \\
\max \left\{\sigma\left(a_{0}\right), \sigma\left(a_{1}\right)\right\}=2=\sigma\left(a_{2}\right), \\
\tau\left(a_{1}\right)=6<\frac{15}{2}=\tau\left(a_{2}\right),
\end{gathered}
$$

where $l=2$ and $\max \left\{p_{2}, p_{1}, p_{0}\right\}=1$. Here $\sigma(g)=$ $3>2=\sigma\left(a_{2}\right)$.

Case 5. If $\sigma(F)>\sigma\left(a_{l}\right)$, then $g(z)$ satisfies (7), where $F(z)=\mathrm{e}^{z^{3}}, a_{2}(z)=\mathrm{e}^{-\left(9 \pi z^{2}+27 \pi^{2} z+27 \pi^{3}\right)} \cot z$, $a_{1}(z)=\mathrm{e}^{-\left(6 \pi z^{2}+12 \pi^{2} z+8 \pi^{3}\right)}$ and $a_{0}(z)=-1$. Then, $a_{j}(z), j=0,1,2$ and $F(z)$ satisfy

$$
\sigma(F)=3>2=\max \left\{\sigma\left(a_{0}\right), \sigma\left(a_{1}\right)\right\}=\sigma\left(a_{2}\right),
$$

where $l=2$ and $\max \left\{p_{2}, p_{1}, p_{0}\right\}=1$. Here $\sigma(g)=$ $\sigma(F)=3$. Moreover, $a_{j}(z), j=0,1,2$ satisfy

$\lambda\left(\frac{1}{a_{2}}\right)=1<2=\sigma\left(a_{2}\right)$ and $\tau\left(a_{1}\right)=6<9=\tau\left(a_{2}\right)$,

these two conditions are not necessary for Case 5.

\section{AUXILIARY LEMMAS}

Cherry and Ye [21] showed the following BorelNevanlinna Growth Lemma.

Lemma 1 ([21]) Let $F(r)$ and $\phi(r)$ be positive, nondecreasing, continuous functions defined for $r_{0} \leqslant r<$ $\infty$, and assume that $F(r) \geqslant$ e for $r \geqslant r_{0}$. Let $\xi(x)$ be a positive, non-decreasing, continuous function defined for $\mathrm{e} \leqslant x<\infty$. Let $C>1$ be a constant, and let $E$ be the closed subset of $\left[r_{0}, \infty\right)$ defined by

$$
E=\left\{r \in\left[r_{0}, \infty\right): F\left(r+\frac{\phi(r)}{\xi(F(r))}\right) \leqslant C F(r)\right\} .
$$

Then, for all $R<\infty$,

$$
\int_{E \cap\left[r_{0}, R\right]} \frac{\mathrm{d} r}{\phi(r)} \leqslant \frac{1}{\xi(\mathrm{e})}+\frac{1}{\log C} \int_{\mathrm{e}}^{F(R)} \frac{\mathrm{d} x}{x \xi(x)} .
$$

The following lemma is an analogue of the the lemma on the logarithmic derivative for meromorphic functions composed with polynomials.
Lemma 2 ([10]) Let $f$ be a meromorphic function such that $f(0) \neq 0, \infty$, let $n \in \mathbb{N}$, and let $\alpha>1$ and $0<\delta<1$. If the polynomials $\omega(z)=c z^{n}+p_{n-1} z^{n-1}+$ $\cdots+p_{0}$ and $\varphi(z)=c z^{n}+q_{n-1} z^{n-1}+\cdots+q_{0}$ are distinct and non-constant, then there exists an $r_{0}>0$ such that, for all $r=|z| \geqslant r_{0}$,

$$
\begin{aligned}
m\left(r, \frac{f \circ \omega}{f \circ \varphi}\right) \leqslant & \frac{K(\alpha, \delta, \omega, \varphi)}{r^{\delta / n}} \times \\
& \left(T\left(\alpha|c| r^{n}, f\right)+\log ^{+} \frac{1}{|f(0)|}\right),
\end{aligned}
$$

where

$$
K(\alpha, \delta, \omega, \varphi)=\frac{8 \alpha C(\delta(\alpha+1)+n(6 \alpha+2))}{\delta(1-\delta)|c|^{\delta / n}(\alpha-1)}
$$

with $C=1+\left|p_{n-1}\right|+\left|q_{n-1}\right|$.

Lemma 3 ([22]) Let $f$ be a meromorphic function with iterated order $0<\sigma_{p}(f)<\infty$ and iterated type $0<\tau_{p}(f)<\infty$. Then for any given $\beta<\tau_{p}(f)$, there exists a subset $E$ of $[1, \infty)$ that has infinite logarithmic measure, such that $\log _{p-1} T(r, f)>\beta r^{\sigma_{p}(f)}$ holds for all $r \in E$.

Chiang and Feng [5] proved that, for any arbitrary $c \in \mathbb{C}$, the Nevanlinna characteristic of any meromorphic function $f$ of finite order satisfies the asymptotic relation $T(r, f(z+c)) \sim T(r, f)(r \rightarrow \infty)$. The following lemma is a generalization of their result to a certain type of composite meromorphic functions, including a class of infinite order functions.

Lemma 4 ([10]) Let $w(z)=c z^{n}+p_{n-1} z^{n-1}+\cdots+p_{0}$ be a non-constant polynomial. If $f$ is a meromorphic function such that

$$
\limsup _{r \rightarrow \infty} \frac{\log \log T(r, f)}{\log r}<\frac{1}{n^{2}},
$$

then

$$
T(r, f \circ w)=(1+o(1)) T\left(|c| r^{n}, f\right),
$$

where $r$ approaches infinity outside a possible exceptional set of finite logarithmic measure.

\section{Proof of Theorem 1}

Proof: It may be helpful to mention that similar techniques (but with more complexity) can be found in [10], Lemma 2.2, and references therein.

Denote $g(r):=T\left(|c| r^{n}, f\right)$ and $\alpha=\beta^{n}$. For positive, non-decreasing, continuous function $\xi(x)$ 
and $\phi(r)$ defined for $\mathrm{e} \leqslant x<\infty$ and $r_{0} \leqslant r<\infty$, respectively, where $r_{0}$ is such that $g(r) \geqslant e$ for all $r \geqslant r_{0}$, Lemma 1 implies that

$$
g\left(r+\frac{\phi(r)}{\xi(g(r))}\right) \leqslant 2 g(r)
$$

for all $r$ outside a set $E$ satisfying

$$
\int_{E \cap\left[r_{0}, R\right]} \frac{\mathrm{d} r}{\phi(r)} \leqslant \frac{1}{\xi(\mathrm{e})}+\frac{1}{\log 2} \int_{e}^{g(R)} \frac{\mathrm{d} x}{x \xi(x)},
$$

where $R<\infty$. By choosing $\phi(r)=r$ and $\xi(x)=$ $(\log (x))^{1+\varepsilon_{1}}$ with $\varepsilon_{1}>0$, and defining

$$
\beta=1+\frac{1}{(\log g(r))^{1+\varepsilon_{1}}},
$$

it follows that

$$
T\left(\alpha|c| r^{n}, f\right)=g(\beta r) \leqslant 2 g(r)=2 T\left(|c| r^{n}, f\right)
$$

for all $r$ outside a set $E$ of finite logarithmic measure. Moreover, by substituting $\alpha=\beta^{n}$ into (8), it follows that there exists a positive absolute constant $C$ such that

$$
K(\alpha, \delta, \omega, \varphi) \leqslant C\left(\log T\left(|c| r^{n}, f\right)\right)^{1+\varepsilon_{1}}
$$

for all $r$ sufficiently large.

Suppose that $\sigma_{2}(f)=\sigma_{2}<1 / n^{2}$, then by the definition of $\sigma_{2}(f)$, for any given $\varepsilon_{2}>0$ and all $r$ large enough, we have $\log T(r, f) \leqslant r^{\sigma_{2}+\varepsilon_{2}}$. Hence, by choosing $\varepsilon_{1}$ sufficiently small in (10), it follows that

$$
K(\alpha, \delta, \omega, \varphi) \leqslant C r^{n\left(\sigma_{2}+\varepsilon_{2}\right)}
$$

for all $r$ sufficiently large. The assertion follows in the case $f(0) \neq 0, \infty$ by choosing $\delta=1-\varepsilon_{2}$ in (8) and combining inequalities (9) and (11). If $f$ has either a zero or pole at the origin, then, for a suitable $k \in \mathbb{Z}$, we may write $f(z)=z^{k} g(z)$ where $g(z)$ is finite and non-zero at the origin. Hence, by choosing $\varepsilon=\varepsilon\left(n, \varepsilon_{2}\right)$ sufficiently small, it follows that

$$
\begin{aligned}
m\left(r, \frac{f \circ \omega}{f \circ \varphi}\right) & \leqslant m\left(r, \frac{g \circ \omega}{g \circ \varphi}\right)+O(\log r) \\
& =o\left(\frac{T\left(|c| r^{n}, g\right)}{r^{\frac{1}{n}-n \sigma_{2}-\varepsilon}}\right)+O(\log r) \\
& =o\left(\frac{T\left(|c| r^{n}, f\right)}{r^{\frac{1}{n}-n \sigma_{2}-\varepsilon}}\right)+O(\log r)
\end{aligned}
$$

outside an exceptional set $E^{\prime}$ of finite logarithmic measure. Therefore, since $f$ is a non-rational meromorphic function, we have

$$
m\left(r, \frac{f \circ \omega}{f \circ \varphi}\right)=o\left(\frac{T\left(|c| r^{n}, f\right)}{r^{\frac{1}{n}-n \sigma_{2}-\varepsilon}}\right)
$$

as $r$ approaches infinity outside $E^{\prime}$. This completes the proof of Theorem 1.

\section{Proof of Theorem 2}

Proof: Let $f(z)(\not \equiv 0)$ be a transcendental meromorphic solution of (5). Suppose that $\sigma(f)<\sigma\left(a_{l}\right) / n+$ $1 / n^{2}$, then by Theorem 1 and choosing $\varepsilon$ sufficiently small, there exists a set $E_{1}$ with finite logarithmic measure such that for all $r \notin E_{1}$ and $r \rightarrow \infty$, we have for $j, l \in\{0,1, \ldots, k\}$

$$
m\left(r, \frac{f \circ P_{j}}{f \circ P_{l}}\right)=o\left(\frac{T\left(|c| r^{n}, f\right)}{r^{\frac{1}{n}-\varepsilon}}\right) .
$$

By the assumptions of Theorem 2, we know that $\sigma\left(a_{l}\right)=\sigma$. For any given sufficiently small $\delta>0$, there exists a set $H_{1}$ satisfying $\overline{\operatorname{dens}}\{r=|z|: z \in$ $\left.H_{1}\right\}>0$ such that for all $z \in H_{1}$ and $|z|=r \rightarrow \infty$, we obtain for $j=0,1, \ldots, k, j \neq l$

$$
m\left(r, a_{j}(z)\right) \leqslant \alpha r^{\sigma-\delta}
$$

We divide (5) by $f\left(P_{l}(z)\right)$ to get

$$
-a_{l}(z)=\sum_{j=0, j \neq l}^{k} a_{j}(z) \frac{f\left(P_{j}(z)\right)}{f\left(P_{l}(z)\right)} .
$$

Now, we may choose sufficiently small $\varepsilon^{\prime}$ satisfying $0<2 \varepsilon^{\prime}<\min \left\{\varepsilon, \delta, n \sigma\left(a_{l}\right)-n^{2} \sigma(f)+1\right\}$, and deduce from (12)-(14) that for sufficiently large $r$ satisfying $|z|=r \in H_{1} \backslash E_{1}$, it follows that

$$
\begin{aligned}
T\left(r, a_{l}\right)=m\left(r, a_{l}\right) \leqslant & \sum_{j=0, j \neq l}^{k} m\left(r, a_{j}\right) \\
& +\sum_{j=0, j \neq l}^{k} m\left(r, \frac{f\left(P_{j}\right)}{f\left(P_{l}\right)}\right)+O(1) \\
\leqslant & O\left(\alpha r^{\sigma-\delta}\right)+o\left(\frac{T\left(|c| r^{n}, f\right)}{r^{\frac{1}{n}-\varepsilon^{\prime}}}\right) \\
= & o\left(r^{\sigma\left(a_{l}\right)-\varepsilon^{\prime}}\right)+o\left(r^{n \sigma(f)-\frac{1}{n}+\varepsilon^{\prime}}\right) \\
= & o\left(r^{\sigma\left(a_{l}\right)-\varepsilon^{\prime}}\right),
\end{aligned}
$$

a contradiction. Hence, we have $\sigma(f) \geqslant \sigma\left(a_{l}\right) / n+$ $1 / n^{2}$. This completes the proof of Theorem 2 .

\section{Proof of Theorem 3}

Proof: Let $f(z)(\not \equiv 0)$ be a transcendental meromorphic solution of (5). Suppose that $\sigma(f)<\sigma\left(a_{l}\right) / n+$ $1 / n^{2}$. We divide through (5) by $f\left(P_{l}(z)\right)$ to get (14). 
It follows that

$$
\begin{aligned}
& T\left(r, a_{l}\right)=m\left(r, a_{l}\right)+N\left(r, a_{l}\right) \\
& \leqslant \sum_{j=0, j \neq l}^{k} m\left(r, a_{j}\right)+\sum_{j=0, j \neq l}^{k} m\left(r, \frac{f\left(P_{j}\right)}{f\left(P_{l}\right)}\right)+N\left(r, a_{l}\right)+O(1) \\
& \leqslant \sum_{j=0, j \neq l}^{k} T\left(r, a_{j}\right)+O\left(m\left(r, \frac{f\left(P_{j}\right)}{f\left(P_{l}\right)}\right)\right)+N\left(r, a_{l}\right)
\end{aligned}
$$

By Theorem 1, there exists a set $E_{2}$ with finite logarithmic measure such that for all $r \notin E_{2}$ and $r \rightarrow \infty$, we have (12).

Let us choose $\alpha_{1}$ such that $\lambda\left(\frac{1}{a_{l}}\right)<\alpha_{1}<\sigma\left(a_{l}\right)=$ $\sigma$. Then for any given $\varepsilon_{1}$ such that $0<2 \varepsilon_{1}<$ $\min \left\{\alpha_{1}-\lambda\left(\frac{1}{a_{l}}\right), \sigma-\alpha_{1}\right\}$ and sufficiently large $r$,

$$
N\left(r, a_{l}\right) \leqslant r^{\lambda\left(\frac{1}{a_{l}}\right)+\varepsilon_{1}}<r^{\alpha_{1}} .
$$

Assume that $\max _{0 \leqslant j \leqslant k, j \neq l}\left\{\sigma\left(a_{j}\right)\right\} \leqslant \sigma\left(a_{l}\right)$ and $\sum_{\sigma\left(a_{j}\right)=\sigma} \tau\left(a_{j}\right)<\tau\left(a_{l}\right)<\infty$. Then there exists a set $J_{1} \subseteq\{0,1, \ldots, l-1, l+1, \ldots, k\}$ such that for $j \in$ $J_{1}$, we have $\sigma\left(a_{j}\right)=\sigma\left(a_{l}\right)=\sigma$ and $\sum_{j \in J_{1}} \tau\left(a_{j}\right)<$ $\tau\left(a_{l}\right)=\tau$. For $i \in\{0,1, \ldots, l-1, l+1, \ldots, k\} \backslash J_{1}$ we have $\sigma\left(a_{i}\right)<\sigma\left(a_{l}\right)=\sigma$. Hence, we can choose $\beta_{1}, \beta_{2}, \sigma_{0}$ satisfying $\sum_{j \in J_{1}} \tau\left(a_{j}\right)=\tau_{0}<\beta_{1}<\beta_{2}<\tau$, $0<\sigma_{0}<\sigma$ such that for any given $\varepsilon_{2}$ such that $0<2 \varepsilon_{2}<\min \left\{\beta_{2}-\beta_{1}, \tau-\beta_{2}, \beta_{1}-\tau_{0}, \sigma-\sigma_{0}, \sigma_{0}\right\}$ and for sufficiently large $r$, we have

$$
T\left(r, a_{j}\right) \leqslant\left(\tau\left(a_{j}\right)+\varepsilon_{2}\right) r^{\sigma}, \quad j \in J_{1},
$$

and for $i \in\{0,1, \ldots, l-1, l+1, \ldots, k\} \backslash J_{1}$

$$
T\left(r, a_{i}\right) \leqslant r^{\sigma_{0}} .
$$

By applying Lemma 3, there exists a subset $E_{3}$ of $[1, \infty)$ that has infinite logarithmic measure such that for all $r \in E_{3}$ and $r \rightarrow \infty$, the inequality

$$
T\left(r, a_{l}\right)>\beta_{2} r^{\sigma}
$$

holds. Now, we may choose sufficiently small $\varepsilon^{\prime}$ satisfying $0<(k+1) \varepsilon^{\prime}<\min \left\{\varepsilon_{1}, \varepsilon_{2}\right\}$, and deduce from (12) and (15)-(19) that for $r \in E_{3} \backslash E_{2}$ and $r \rightarrow \infty$, we have

$$
\begin{aligned}
\beta_{2} r^{\sigma}< & \sum_{j \in J_{1}}\left(\tau\left(a_{j}\right)+\varepsilon^{\prime}\right) r^{\sigma}+\sum_{i \in\{0,1, \ldots, l-1, l+1, \ldots, k\} \backslash J_{1}} r^{\sigma_{0}} \\
& +o\left(\frac{T\left(|c| r^{n}, f\right)}{r^{\frac{1}{n}-\varepsilon^{\prime}}}\right)+r^{\alpha_{1}} \\
< & \left(\beta_{1}+k \varepsilon^{\prime}\right) r^{\sigma}+O\left(r^{\sigma_{0}}\right)+o\left(r^{n \sigma(f)-\frac{1}{n}+\varepsilon^{\prime}}\right)+r^{\alpha_{1}} .
\end{aligned}
$$

It follows that

$$
\left(\beta_{2}-\beta_{1}-k \varepsilon^{\prime}\right) r^{\sigma}<O\left(r^{\sigma_{0}}\right)+o\left(r^{n \sigma(f)-\frac{1}{n}+\varepsilon^{\prime}}\right)+r^{\alpha_{1}} .
$$

Since $0<(k+1) \varepsilon^{\prime}<\min \left\{\varepsilon_{1}, \varepsilon_{2}\right\}$, we obtain from (20) that $\sigma\left(a_{l}\right)=\sigma \leqslant n \sigma(f)-1 / n$. Hence, we have $\sigma(f) \geqslant \sigma\left(a_{l}\right) / n+1 / n^{2}$. This completes the proof of Theorem 3.

\section{Proof of Theorem 4}

Proof: Let $f(z)(\not \equiv 0)$ be a meromorphic solution of (6). Note that $m=\max \left\{p_{j}\right\}, j=0,1, \ldots, k$. We divide through (6) by $f\left(P_{l}(z)\right)$ to get

$$
-a_{l}(z)=\sum_{j=0, j \neq l}^{k} a_{j}(z) \frac{f\left(P_{j}(z)\right)}{f\left(P_{l}(z)\right)}-\frac{F(z)}{f\left(P_{l}(z)\right)} .
$$

It follows that

$$
\begin{aligned}
T\left(r, a_{l}\right)= & m\left(r, a_{l}\right)+N\left(r, a_{l}\right) \\
\leqslant & \sum_{j=0, j \neq l}^{k} m\left(r, a_{j}\right)+\sum_{j=0, j \neq l}^{k} m\left(r, \frac{f\left(P_{j}\right)}{f\left(P_{l}\right)}\right) \\
& +m(r, F)+m\left(r, \frac{1}{f\left(P_{l}\right)}\right)+N\left(r, a_{l}\right)+O(1) \\
\leqslant & \sum_{j=0, j \neq l}^{k} T\left(r, a_{j}\right)+O\left(\sum_{j=0}^{k} T\left(r, f\left(P_{j}\right)\right)\right)+T(r, F)+N\left(r, a_{l}\right) .
\end{aligned}
$$

Lemma 4 implies that there exists a set $E_{4}$ with finite logarithmic measure such that for all $r \notin E_{4}$ and $r \rightarrow \infty$, we have

$$
\begin{aligned}
T\left(r, f\left(P_{j}(z)\right)\right) & =(1+o(1)) T\left(\left|c_{j p_{j}}\right| r^{p_{j}}, f\right) \\
& \leqslant(1+o(1)) T\left(c r^{p_{j}}, f\right) \\
& \leqslant(1+o(1)) T\left(c r^{m}, f\right),
\end{aligned}
$$

where $c=\max \left\{\left|c_{0 p_{0}}\right|,\left|c_{1 p_{1}}\right|, \ldots,\left|c_{k p_{k}}\right|\right\}$.

Let us choose $\alpha_{2}$ such that $\lambda\left(\frac{1}{a_{l}}\right)<\alpha_{2}<\sigma\left(a_{l}\right)=$ $\sigma$. Then for any given $\varepsilon_{1}$ such that $0<2 \varepsilon_{1}<$ $\min \left\{\alpha_{2}-\lambda\left(\frac{1}{a_{l}}\right), \sigma-\alpha_{2}\right\}$ and sufficiently large $r$,

$$
N\left(r, a_{l}\right) \leqslant r^{\lambda\left(\frac{1}{a_{l}}\right)+\varepsilon_{1}}<r^{\alpha_{2}} .
$$

Assume that $\max _{0 \leqslant j \leqslant k, j \neq l}\left\{\sigma\left(a_{j}\right)\right\} \leqslant \sigma\left(a_{l}\right)$ and $\sum_{\sigma\left(a_{j}\right)=\sigma} \tau\left(a_{j}\right)<\tau\left(a_{l}\right)<\infty$. Then there exists a set $J_{2} \subseteq\{0,1, \ldots, l-1, l+1, \ldots, k\}$ such that for $j \in$ $J_{2}$, we have $\sigma\left(a_{j}\right)=\sigma\left(a_{l}\right)=\sigma$ and $\sum_{j \in J_{2}} \tau\left(a_{j}\right)<$ $\tau\left(a_{l}\right)=\tau$ and for $i \in\{0,1, \ldots, l-1, l+1, \ldots, k\} \backslash J_{2}$ we have $\sigma\left(a_{i}\right)<\sigma\left(a_{l}\right)=\sigma$. Hence, we can choose $\beta_{3}, \beta_{4}, \sigma^{\prime}$ satisfying $\sum_{j \in J_{2}} \tau\left(a_{j}\right)=\tau_{1}<\beta_{3}<\beta_{4}<\tau$, $0<\sigma^{\prime}<\sigma$ such that for any given $\varepsilon_{2}$ such that $0<2 \varepsilon_{2}<\min \left\{\beta_{4}-\beta_{3}, \tau-\beta_{4}, \beta_{3}-\tau_{1}, \sigma-\sigma^{\prime}, \sigma^{\prime}\right\}$ and sufficiently large $r$, the inequalities

$$
T\left(r, a_{j}\right) \leqslant\left(\tau\left(a_{j}\right)+\varepsilon_{2}\right) r^{\sigma}, \quad j \in J_{2}
$$


and for $i \in\{0,1, \ldots, l-1, l+1, \ldots, k\} \backslash J_{2}$

$$
T\left(r, a_{i}\right) \leqslant r^{\sigma^{\prime}}
$$

hold. By applying Lemma 3 , there exists a subset $E_{5}$ of $[1, \infty)$ that has infinite logarithmic measure such that for all $r \in E_{5}$ and $r \rightarrow \infty$, we have

$$
T\left(r, a_{l}\right)>\beta_{4} r^{\sigma}
$$

Case 1. If $\sigma(F)<\sigma\left(a_{l}\right)$, we can choose $\alpha_{3}$ such that $\sigma(F)<\alpha_{3}<\sigma\left(a_{l}\right)=\sigma$. Then for any given $\varepsilon_{3}$ such that $0<2 \varepsilon_{3}<\min \left\{\alpha_{3}-\sigma(F), \sigma-\alpha_{3}\right\}$ and sufficiently large $r$, we obtain

$$
T(r, F) \leqslant r^{\sigma(F)+\varepsilon_{3}}<r^{\alpha_{3}} .
$$

Now, we choose sufficiently small $\varepsilon$ satisfying $0<(k+1) \varepsilon<\min \left\{\varepsilon_{1}, \varepsilon_{2}, \varepsilon_{3}\right\}$, and deduce from (22)-(28) that for $r \in E_{5} \backslash E_{4}$ and $r \rightarrow \infty$, we have

$$
\begin{gathered}
\beta_{4} r^{\sigma}<\sum_{j \in J_{2}}\left(\tau\left(a_{j}\right)+\varepsilon_{2}\right) r^{\sigma}+\sum_{i \in\{0,1, \ldots, l-1, l+1, \ldots, k\} \backslash J_{2}} r^{\sigma_{0}} \\
+O\left(T\left(c r^{m}, f\right)\right)+r^{\alpha_{2}}+r^{\alpha_{3}} \\
<\left(\beta_{3}+k \varepsilon\right) r^{\sigma}+O\left(r^{\sigma^{\prime}}\right)+O\left(r^{m \sigma(f)+\varepsilon}\right)+r^{\alpha_{2}}+r^{\alpha_{3}} .
\end{gathered}
$$

It follows that

$$
\left(\beta_{4}-\beta_{3}-k \varepsilon\right) r^{\sigma}<O\left(r^{\sigma^{\prime}}\right)+O\left(r^{m \sigma(f)+\varepsilon}\right)+r^{\alpha_{2}}+r^{\alpha_{3}} .
$$

Since $0<(k+1) \varepsilon<\min \left\{\varepsilon_{1}, \varepsilon_{2}, \varepsilon_{3}\right\}$, we obtain from (29) that $\sigma\left(a_{l}\right)=\sigma \leqslant m \sigma(f)$. Hence, we have $\sigma(f) \geqslant \sigma\left(a_{l}\right) / \max \left\{p_{j}\right\}$.

If $\sigma(F)=\sigma\left(a_{l}\right)$ and $\sum_{\sigma\left(a_{j}\right)=\sigma\left(a_{l}\right), j \neq l} \tau\left(a_{j}\right)+$ $\tau(F)<\tau\left(a_{l}\right)$, then for the $\varepsilon$ above and sufficiently large $r$, we have

$$
T(r, F) \leqslant(\tau(F)+\varepsilon) r^{\sigma} .
$$

Now, we may choose sufficiently small $\varepsilon$ satisfying $0<(k+2) \varepsilon<\min \left\{\varepsilon_{1}, \varepsilon_{2}, \varepsilon_{3}\right\}$, and deduce from (22)-(27) and (30) that for $r \in E_{5} \backslash E_{4}$ and $r \rightarrow \infty$,

$$
\begin{aligned}
\beta_{4} r^{\sigma}< & \sum_{j \in J_{2}}\left(\tau\left(a_{j}\right)+\varepsilon_{2}\right) r^{\sigma}+\sum_{i \in\{0,1, \ldots, l-1, l+1, \ldots, k\} \backslash J_{2}} r^{\sigma^{\prime}} \\
& +O\left(T\left(c r^{m}, f\right)\right)+r^{\alpha_{2}}+(\tau(F)+\varepsilon) r^{\sigma} \\
< & \left(\beta_{3}+k \varepsilon\right) r^{\sigma}+O\left(r^{\sigma^{\prime}}\right)+O\left(r^{m \sigma(f)+\varepsilon}\right) \\
& +r^{\alpha_{2}}+(\tau(F)+\varepsilon) r^{\sigma} .
\end{aligned}
$$

It follows that

$$
\begin{aligned}
\left(\beta_{4}-\beta_{3}-\tau(F)\right. & -(k+1) \varepsilon) r^{\sigma} \\
& <O\left(r^{\sigma^{\prime}}\right)+O\left(r^{m \sigma(f)+\varepsilon}\right)+r^{\alpha_{2}}
\end{aligned}
$$

Since $0<(k+2) \varepsilon<\min \left\{\varepsilon_{1}, \varepsilon_{2}, \varepsilon_{3}\right\}$, we obtain from (31) that $\sigma\left(a_{l}\right)=\sigma \leqslant m \sigma(f)$. Hence, we have $\sigma(f) \geqslant \sigma\left(a_{l}\right) / \max \left\{p_{j}\right\}$.

If $\sigma(F)=\sigma\left(a_{l}\right)$ and $\sum_{\sigma\left(a_{j}\right)=\sigma\left(a_{l}\right), j \neq l} \tau\left(a_{j}\right)+$ $\tau\left(a_{l}\right)<\tau(F)$. From (6) it follows that for sufficiently large $r$,

$$
\begin{aligned}
& T(r, F) \leqslant \sum_{j=0}^{k} T\left(r, a_{j} f\left(P_{j}\right)\right)+O(1) \\
& \leqslant \sum_{j=0, j \neq l}^{k} T\left(r, a_{j}\right)+O\left(\sum_{j=0}^{n} T\left(r, f\left(P_{j}\right)\right)\right)+T\left(r, a_{l}\right) .
\end{aligned}
$$

Then by Lemma 3, for the $\varepsilon$ above, there exists a subset $E_{6}$ of $[1, \infty)$ that has infinite logarithmic measure such that for all $r \in E_{6}$ and $r \rightarrow \infty$,

$$
T(r, F)>(\tau(F)-\varepsilon) r^{\sigma} .
$$

By the definition of $\tau\left(a_{l}\right)$, we have that for the $\varepsilon$ above and sufficiently large $r$, the inequality

$$
T\left(r, a_{l}\right) \leqslant(\tau+\varepsilon) r^{\sigma}
$$

holds. Now, we may choose sufficiently small $\varepsilon$ satisfying $0<(k+3) \varepsilon<\min \left\{\varepsilon_{1}, \varepsilon_{2}, \varepsilon_{3}\right\}$, and deduce from (23), (25), (26) and (32)-(34) that for $r \in$ $E_{6} \backslash E_{4}$ and $r \rightarrow \infty$, we have

$$
\begin{aligned}
&(\tau(F)-\varepsilon) r^{\sigma}<\sum_{j \in J_{2}}\left(\tau\left(a_{j}\right)+\varepsilon_{2}\right) r^{\sigma}+\sum_{i \in\{0,1, \ldots, l-1, l+1, \ldots, k\} \backslash J_{2}} r^{\sigma^{\prime}} \\
&+O\left(T\left(c r^{m}, f\right)\right)+(\tau+\varepsilon) r^{\sigma} \\
&<\left(\beta_{3}+k \varepsilon\right) r^{\sigma}+O\left(r^{\sigma^{\prime}}\right)+O\left(r^{m \sigma(f)+\varepsilon}\right)+(\tau+\varepsilon) r^{\sigma}
\end{aligned}
$$

It follows that

$$
\left(\tau(F)-\tau-\beta_{3}-(k+2) \varepsilon\right) r^{\sigma}<O\left(r^{\sigma^{\prime}}\right)+O\left(r^{m \sigma(f)+\varepsilon}\right) .
$$

Since $0<(k+3) \varepsilon<\min \left\{\varepsilon_{1}, \varepsilon_{2}, \varepsilon_{3}\right\}$, we obtain from (35) that $\sigma\left(a_{l}\right)=\sigma \leqslant m \sigma(f)$. Hence, we have $\sigma(f) \geqslant \sigma\left(a_{l}\right) / \max \left\{p_{j}\right\}$.

Case 2. If $F(z) \equiv 0$, then by using a similar reasoning as the one in Theorem 3 , we have $\sigma(f) \geqslant$ $\sigma\left(a_{l}\right) / \max \left\{p_{j}\right\}$.

Case 3. If $\sigma(F)>\sigma\left(a_{l}\right)$, then we may suppose that $\sigma(f)<\sigma(F) / \max \left\{p_{j}\right\}$ on the contrary. By (6) and Lemma 4, we obtain

$$
\sigma\left(a_{k}(z) f\left(P_{k}(z)\right)+\cdots+a_{0}(z) f\left(P_{0}(z)\right)\right)<\sigma(F),
$$

a contradiction. Hence, we have $\sigma(f) \geqslant$ $\sigma(F) / \max \left\{p_{j}\right\}$. This completes the proof of Theorem 4 . 
Acknowledgements: This work is supported by the National Natural Science Foundation of China (No. 11901111) and Youth innovation talents program of Guangdong provincial department of education (2016WQNCX046). Part of this work was done during the authors visit to the Chern Institute of Mathematics at Nankai University, and the authors would like to appreciate their hospitality.

\section{REFERENCES}

1. Laine I (1993) Nevanlinna Theory and Complex Differential Equations, Walter de Gruyter, Berlin.

2. Gromak VI, Laine I, Shimomura S (2002) Painlevé Differential Equations in the Complex Plane, Walter de Gruyter, Berlin.

3. Halburd RG, Korhonen RJ (2006) Difference analogue of the lemma on the logarithmic derivative with applications to difference equations. $J$ Math Anal Appl 314, 477-487.

4. Halburd RG, Korhonen RJ (2007) Finite-order meromorphic solutions and the discrete Painlevé equations. Proc Lond Math Soc 94, 443-474.

5. Chiang YM, Feng SJ (2008) On the Nevanlinna characteristic of $f(z+\eta)$ and difference equations in the complex plane. Ramanujan J 16, 105-129.

6. Halburd RG, Korhonen RJ, Tohge K (2014) Holomorphic curves with shift-invariant hyperplane preimages. Trans Amer Math Soc 366, 4267-4298.

7. Hayman WK (1964) Meromorphic Functions, Clarendon Press, Oxford.

8. Yi HX, Yang CC (2003) The Uniqueness Theory of Meromorphic Functions, Kluwer Academic Publishers, Dordrecht.

9. Sato D (1963) On the rate of growth of entire functions of fast growth. Bull Amer Math Soc 69, 411-414.
10. Korhonen R (2009) An extension of Picard's theorem for meromorphic functions of small hyper-order. $J$ Math Anal Appl 357, 244-253.

11. Laine I, Yang CC (2007) Clunie theorems for difference and q-difference polynomials. $J$ Lond Math Soc 76, 556-566.

12. Korhonen R, Wen ZT (2016) Existence of zero-order meromorphic solutions in detecting $q$-difference Painlevé equations. Trans Amer Math Soc 368, 4993-5008.

13. Wen ZT (2016) Finite order solutions of difference equations, and difference Painlevé equations IV. Proc Amer Math Soc 144, 4247-4260.

14. Wen ZT, Ye Z (2016) Wiman-Valiron theorem for $q$ differences. Ann Acad Sci Fenn Math 41, 305-312.

15. Zheng XM, Tu J (2011) Growth of meromorphic solutions of linear difference equations. $J$ Math Anal Appl 384, 349-356.

16. Liu K, Qi XG (2011) Meromorphic solutions of $q$-shift difference equation. Ann Polon Math 101, 215-225.

17. Liu HF, Mao ZQ (2013) On the meromorphic solutions of some linear difference equations. Adv Differ Equ 133, 1-12.

18. Huang ZB, Zhang RR (2018) Properties on qdifference Riccati equation. Bull Korean Math Soc 55, 1755-1771.

19. Rong JX, Xu JF (2019) Three results on the nonlinear differential equations and differential-difference equations. Mathematics 7, 1-11.

20. Du YF, Gao ZS, Zhang JL, Zhao M (2018) Value distribution of meromorphic solutions of some second order equations. ScienceAsia 44, 218-225.

21. Cherry W, Ye Z (1993) Nevanlinna's Theory of Value Distribution, Springer-Verlag, Berlin.

22. Cao TB, Xu JF, Chen ZX (2010) On the mermorphic solutions of linear differential equations on the complex plane. J Math Anal Appl 364, 130-142. 